\title{
Application of occupancy and booking information to optimize space and energy use in higher education institutions
}

\author{
Shoaib Azizi*, Ramtin Rabiee, Gireesh Nair, and Thomas Olofsson \\ Department of Applied Physics and Electronics, Umeå University, 90187 Umeå, Sweden
}

\begin{abstract}
Building in higher education institutions (HEIs) are characterized as energy intensive Experience from practice showing large discrepancies between predicted and actual energy performance. The deviation can sometimes reach $100 \%$. Explanations often originate from occupant's behaviour along with building operation. IoT-based smart tools can provide extensive information about building usage to improve the building management, often associates with opportunities, for significant energy saving. The analysis in this study investigate space use based on occupancy and booking information in eight different lecture rooms at Umeå University. The results suggest interventions for significant saving potentials, such as modification of access permission and closing down redundant lecture rooms.
\end{abstract}

\section{Introduction}

Emerging information technologies can offer cost savings and environmental sustainability to the building sector through novel management and control strategies. Internet of things (IoT) is the integration of several technologies to provide smart services in smart environments [1]. Applications of IoT in academic environments associate with the concept of 'smart campus' can improve the student learning experience, campus security and also operational efficiency [2]. A common application of such systems in universities is to support users to find available spaces. While in other industries, its application is relatively more targeted towards efficient use of space [3].

Optimum space management means the space is being used for the maximum time while minimizing the operation and maintenance costs. Space management can prevent squandering of space that lead to attractive return on investment. Optimization of space use may lead to direct savings on energy and other services in addition to preventing the unnecessary new constructions. Moreover, the information of space utilization is useful for strategic planning to identify demand for certain type of spaces [4].

The objective of this study is to investigate the possible applications of IoT related technologies to optimize the space use management for the lecture rooms in universities. This study is an effort to maximize the effective utilization of lecture rooms by modifications to their operation management. The results may indicate the need for new spaces and facilitate the planning for future developments. On the other hand, the optimum space management may enable to close down some lecture rooms thereby reducing the rental cost for university or allow the lecture rooms to be leased to outsiders to generate additional revenue for the building manager.

The hypothesis in this study is that there is a potential to reduce energy use by space-use optimization in university buildings. The data collected from smart tools are used to support the university's organisational objectives of reducing energy and carbon footprint. This study explores the possibility to space management and the analysis is restricted to a few lecture rooms in a university building.

\subsection{Space and energy management in HEls}

Higher education institutions (HEI) have had rapid growth in recent years. Since 1990 the number of these institutions have almost doubled worldwide [5]. The growth is more likely to continue in future due to evolving economic needs and social aspirations [6]. Physical environment is an important aspect of a higher education institutions impacting their performance [7]. Usually, the real estate costs, such as acquisition, construction, maintenance and use constitute the highest cost after staff salaries in universities [8]. Further, the need for efficiency and sustainability requires the universities to explore new ways to better use their resources.

Energy use in non-residential buildings such as university buildings is expected to increase $57 \%$ from

*Corresponding author: shoaib.azizi@umu.se 
2018 to 2050 [9]. The energy intensity in the Swedish university buildings managed by Akademiska Hus is equal to $201.4 \mathrm{kWh} / \mathrm{sqm}-\mathrm{yr}$ [10]. Energy use in this sector is complex due to diversity of activities and type of spaces such as lecture rooms, laboratories and offices $[11,12]$. Moreover, the occupants of such buildings may not be motivated to reduce the energy use since they are usually not aware of the energy costs and also may perceive the energy to be free[13].

Improvement in the operation of educational institutions have driven many research studies on efficient space use by focusing on the booking systems for courses and exams in universities. However, there is a gap between the theoretical approaches and practical applications by education administrators [14]. Moreover, the gap between predicted (timetabled) and actual use in terms of frequency, occupancy and usage by the right group of students is a shortcoming of booking systems [3]. Space utilization surveys have been undertaken by direct observation in small areas [15], however, this method could be impractical for large spaces for longer durations. Optimizing space use is impossible without good data to make decisions. The difficulty to accurately measure the space utilization in buildings have often caused the potential optimization of space use to be ignored [16].

The "Smart Campus Tools" project in Netherland investigates the potentials for space use optimization in academic environments. They define a smart tool as a service or product with which information on space use is collected real-time to improve utilization of the current campus on the one hand, and to improve decisionmaking about the future campus on the other hand [3]. In this view, a monitoring system with PIR (passive infrared radiation) sensors and a booking system fall in the category of smart tools. Their definition of smart tool can be considered as an application of IoT to create a smart environment, which resonate the approach in this study. However, to the best of authors' knowledge, there are no empirical study addressing the space use optimization by using the combination of booking and occupancy information.

According to [17], space utilization can be determined by frequency rate and occupancy rate. Frequency rate is the proportion of time that space is used compared to its availability. Occupancy rate indicates how full the space is compared to its capacity. Online booking systems are widely used in different universities to support users to reserve their required spaces. Beyond supporting the users to organize their activities, these systems can provide information on space use. Moreover, this information can be crossverified by linking the booking systems to sensor technologies to measure the actual usage of spaces. Occupancy based building automation technologies can mitigate the negative effects of occupancy behaviour and poor building control on energy use [18].

Research on IoT tools in buildings has been more technology-oriented rather than involving different disciplines related to building industry to identify the added value of these systems [19]. Majority of papers on the subject focus on performance criteria such as accuracy for a new system design applied in a small scale [20]. The prominent potential that IoT and big data offer may fundamentally change the operation and management of real-estate in universities [21].

\section{Data collection}

The data for this study was collected from 71 wireless sensor devices in 8 different lecture rooms in a building in Umeå University in northern Sweden. Each sensor device could measure PIR, temperature, humidity and light intensity although for the analysis in this study only the PIR data was used. Frequency of data logging was restricted by factors such as capacity of data storage and battery life. The sensors were set to transmit data in 10 minutes intervals using LoRa (Long Range) wireless network which is a low-power wide-area network technology gained fast-paced growth in IoT applications [22]. The data was collected in a middleware database platform which allows easy access to all sensors. This analysis uses data collected during a 4 months period in the spring semester 2019. The number of sensors in each lecture room vary due to difference in size of each lecture room. The installation of sensors in the lecture rooms enable to measure the proportion of time that space is used in relation to availability, the distribution of occupancy in each lecture room and also occupancy rate. To investigate the occupancy rate, the sensors are tried to be distributed evenly, however, since the number of sensors and limitations for sensor installations are different in each lecture room, the comparison of occupancy rate between different lecture rooms is unreliable. Instead, the occupancy rates are compared for each lecture room for different periods.

The sensors might be triggered due to heated surfaces (e.g. by sunlight) or they may fail to detect if the occupants remain overly static. The detection accuracy of sensors were tested in 3 offices for 5 working days. For this trial the occupants were asked to record "ground-truth" information about their presence in the offices. This information was then cross verified with the occupancy detection data from the sensors. The results show that the average accuracy was $95 \%$ when detection occurs within 2 meters. Such uncertainties in the data cannot be excluded though the occurrence of such events that are infrequent and the detections are accurate enough for the analysis.

The room-booking system in Umeå University known as "TimeEdit" allows the university staff to book the lecture rooms. The system is based on a centralized coordinated timetable that allows scheduling the lectures and meetings in a desired lecture room. The system has a user-friendly interface that also presents the features of lecture room such as occupant capacity and available equipment. The information from this booking system 
along with the deployed sensors were used to investigate the usage patterns of the studied lecture rooms.

The investigated lecture rooms are located in a building which is close to most of the departments in the university campus and are usually booked by the employees for teaching-related activities. All the lecture rooms are located in the same floor, beside each other along a corridor. Their proximity to each other diminishes the importance of their spatial location with respect to employees' booking choice. The lecture rooms are supplied with constant ventilation flow for maximum room capacity set on schedule to operate from $6: 30$ to 18:00. The room temperature is set on $21{ }^{\circ} \mathrm{C}$ while the heating is delivered by a hydronic radiator system. The lighting control varies among different lecture rooms while 3 out of the 8 investigated lecture rooms have manual control and the rest have automatic lighting control based on occupancy detection. The size of lecture rooms are related to their capacity ranging from 20 to 130 persons. As presented in Table 1, the lecture rooms can be divided based on their capacity in different size categories. There are 3 lecture rooms with the capacity lower than 35 persons categorized as "small" and 3 lecture rooms with the capacity between 35 and 70 persons categorized as "medium". The two large lecture rooms have 130 persons capacity. Figure 1 shows the layout of lecture room M1 with 7 PIR sensors. Sensor A is installed to detect presence in front of white board and sensors $B$ to $G$ are installed to cover all sitting areas.

Table 1. Categorization of lecture rooms

\begin{tabular}{|l|l|l|}
\hline Size categories & Capacity (persons) & Lecture rooms \\
\hline Small & "Capacity" $<35$ & S1, S2, S3 \\
\hline Medium & $35<$ "Capacity" $<70$ & M1, M2, M3 \\
\hline Large & "Capacity" $>70$ & L1, L2 \\
\hline
\end{tabular}

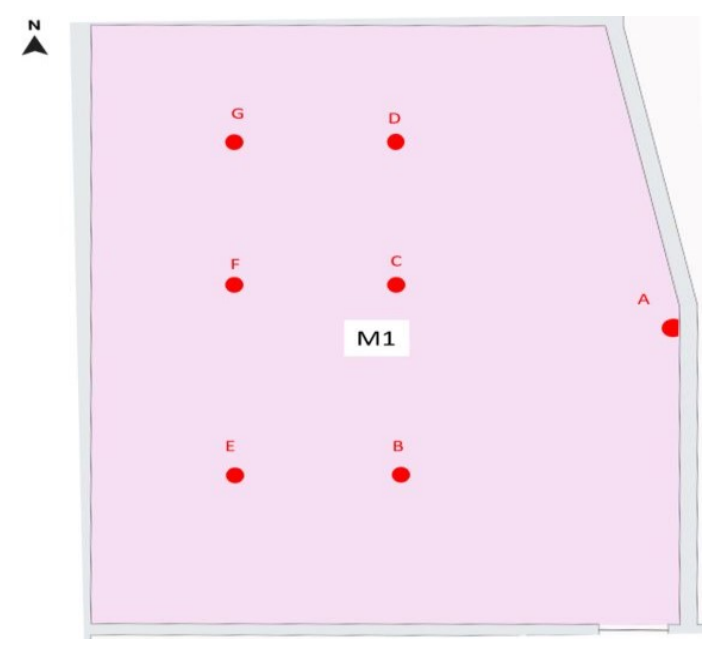

Figure 1. Sensor installation layout in one sample lecture room

\section{Analysis}

As per the objective of this study to optimize the space and energy management, the first step was to study how the lecture rooms are being used. The data was prepared for the analysis in a pre-processing step by MATLAB. Due to interruptions in wireless network there were some missing data from a few sensors. The missing datapoints had to be identified to synchronize (time alignment) different sensors. The data related to weekends and holidays was filtered out and only the time between 8 am and 5 pm during the working days was considered "available period" for the investigation.

As shown in Figure 2, the lecture rooms can be in 4 different statuses considering their booking and occupancy information. The first status is while a lecture room is booked and the occupancy sensors indicate presence during that time (booked-occupied). The second status is when a lecture room is shown to be booked although the occupancy sensors do not detect any presence (booked-unoccupied). The $3^{\text {rd }}$ status is when a lecture room is not booked but the occupancy sensors detect presence as it might be used without prior booking (unbooked-occupied). The last status is related to the period when a lecture room is not booked and not occupied (unbooked-unoccupied). By using the information from booking system and occupancy sensors, the proportion of time related to each status can be calculated implicating the efficiency of space use for each lecture room.

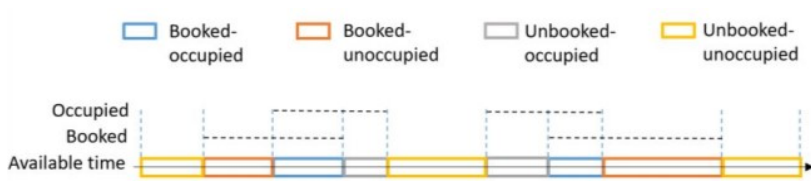

Figure 2. Different statuses based on booking and occupancy information

Calculation of occupancy rate becomes possible by counting the number of occupants present in a lecture room divided by the lecture room's capacity. In this study, the sensor setups with PIR sensors did not allow counting the number of occupants as PIR sensors only detects presence. Nevertheless, when the sensor does not detect presence, the number of occupants is certainly equal to zero. Multiple PIR sensors in each lecture room allow indirect estimation of occupancy rate when they are installed to cover the entire lecture room's area. Presence detection by any installed sensor can infer that lecture room is occupied while some of the sensors might not detect presence. Maximum possible occupancy rate (MPOR) is a measurable parameter by PIR sensors and is defined as the maximum possible number of occupants present in an area (lecture room) in relation to the capacity. For example, if there are 10 sensors installed to cover the entire area in a lecture room while 5 sensors detect presence, and 5 sensors do not, it can be inferred that the maximum number of occupants in that lecture room could not be more than half of the capacity. MPOR can be calculated for each lecture room during a "data acquisition frequency" period. To calculate this parameter for a longer period, MPOR is averaged for the entire periods that the rooms are inferred to be occupied. 
The concept of MPOR that is presented in this study due to limited binary output of PIR sensors, cannot completely replace the regular occupancy rate. The precision and usability of this parameter on describing the occupant distribution depends on how evenly the sensors are installed within each lecture room. This parameter can be calculated only during the periods when the rooms are occupied. These periods consist of two statuses (booked-occupied and unbooked-occupied) while this parameter enables comparison of occupancy and efficient space use between them. Comparison of this parameter between different lecture rooms can be misleading since the number of sensors and their deployment density are different in each lecture room.

Finally, to find out if there is any potential to close down redundant lecture rooms, the study adopts an aggregated approach to examine how often the lecture rooms are booked all together. Such analysis can be undertaken for different periods to check if it is possible to close down a certain lecture room in any period to save energy and other costs.

\section{Results}

\subsection{Statuses}

Figure 3 presents the proportion of possible statuses in relation to the total time (working days from 8 to 17) for the investigated lecture rooms. The proportion of booked periods for these 8 lecture rooms range from $26 \%$ to $80 \%$ with average value of $40 \%$. This indicates that most of times the lecture rooms are not booked. The booked period is shorter for medium sized lecture rooms with $32 \%$ and longer for small sized lecture rooms with $49 \%$. This indicates that small sized lecture rooms are more in need compared to other lecture rooms although even they remain unbooked most of the times.

\subsubsection{Booked-occupied}

This status represents the periods that the lecture rooms are occupied as they were previously planned within the booking system. A high proportion of this status is desired since it signifies efficient use of available rooms. The proportions of duration of this status in relation to the available period for the investigated lecture rooms range from $21 \%$ to $52 \%$ with the average value of $30 \%$. Efforts should be made to increase the duration of this status in the lecture rooms by reducing the duration of other wasteful statuses which on an average stand for $70 \%$ of available times.

\subsubsection{Booked-unoccupied}

The periods that are booked but not occupied may happen due to cancellation of lectures without cancelling the booking of lecture rooms or when a lecture begins later or ends earlier than it was planned. In average, about $9 \%$ of the available time of the lecture rooms are related to this status. The duration of this status has relation with the duration of total booked periods as the lecture rooms that are booked more frequently are likely to have longer duration of this status. Further investigation shows, in average, in $22 \%$ of the periods that the lecture rooms were booked, there was no occupant in the lecture rooms. This value is lower for large lecture rooms with $15 \%$ as compared to other lecture rooms with $22 \%$. Minimizing the duration of this status would foster the optimum use of space in lecture rooms.

\subsubsection{Unbooked-occupied}

This status indicates the periods that the lecture rooms are used in form of drop-in despite not being booked. The users could be the lecturers or staff who had their lectures or meetings without any booking. The other category of users are the students who decide to use the space for studying or group works. The problem with this status is that the lecture rooms might be occupied by fewer number of occupants while the energy services such as lighting, heating and ventilation are provided for the entire room capacity. This is further investigated in the next section by the parameter of MPOR. On average, this status constitutes $19 \%$ of available periods of lecture rooms. During $32 \%$ of the periods that the lecture rooms are not booked, there are occupants using them. This situation is more common for large lecture rooms with $40 \%$ as compared to other lecture rooms with about $29 \%$.

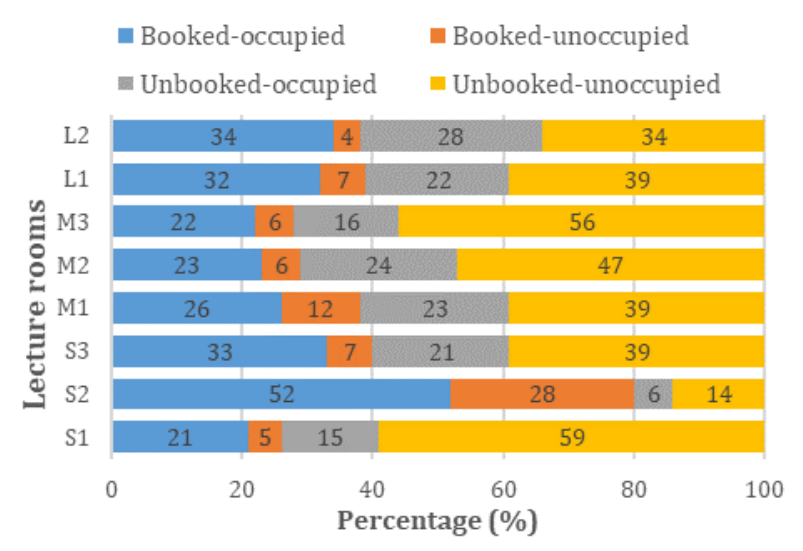

Figure 3. Proportion of different statuses in relation to the total time

\subsubsection{Unbooked-unoccupied}

This status represents the periods that the lecture rooms are neither booked nor occupied therefore is the most obvious case for energy and space optimization. In average, $40 \%$ of available time of lecture rooms (ranging from $14 \%$ to $59 \%$ ) is related to this status. The energy 
savings in this period are relatively easy to achieve by shutting down services.

\subsection{Maximum possible occupancy rate (MPOR)}

MPOR is a parameter that uses the certain unused proportion of the space of a lecture room during the occupied periods to estimate the maximum number of occupants present in a lecture room. Figure 4 presents this average MPOR for the investigated lecture rooms in the two statuses of "booked and occupied" and "unbooked and occupied". The results show this parameter has significantly lower value during the unbooked periods compared to the booked periods while it seems to be independent from size of the lecture rooms. Average MPOR in different lecture rooms during booked periods ranges from $55 \%$ to $75 \%$ with the average value of $67 \%$. During the unbooked periods, the range is from $15 \%$ to $28 \%$ averaged at $22 \%$. These results confirm the previously stated assumption that the lecture rooms are used inefficiently by fewer number of occupants during the unbooked periods and indicates the need for interventions for the status of "unbookedoccupied".
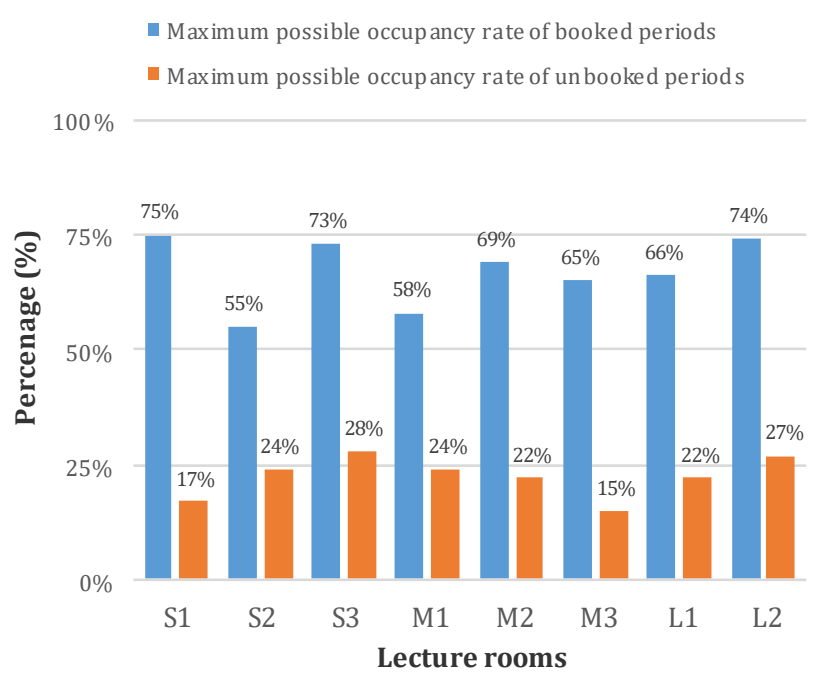

Figure 4. Minimum vacancy rate in the occupied periods of the lecture rooms

\subsection{Aggregation model of space optimization}

The temporal analysis presented in this section aims to establish a method to evaluate the extent that the lecture rooms are needed based on the historical booking information. Although the only status the lecture rooms are being used efficiently is when they are booked and occupied, this analysis includes the period that they are booked but unoccupied. The booked-unoccupied status is considered inevitable and the length of this status is just $9 \%$ of total time in average.

The booking information for all the lecture rooms within similar size category is considered together in an aggregated manner. The results enable to recognize the redundant lecture rooms and the possibility to close them down. On the other hand, the results can also provide reassurance for required new developments by detecting the rooms that are in high demand. The analysis is performed for the typical time slots of lectures in academic environments in Sweden: "8-10", "10-12", "13-15" and "15-17". The time slot "12-13" is often considered lunchtime and is presumably rare to have lectures and meetings at this time. Figure 5 presents the analysis to investigate the proportion of the number of lecture rooms that were booked at the same time. Accordingly, in about only $1 \%$ of the available periods all the lecture rooms are booked at the same time and this happens only in the "10-12" time slot.

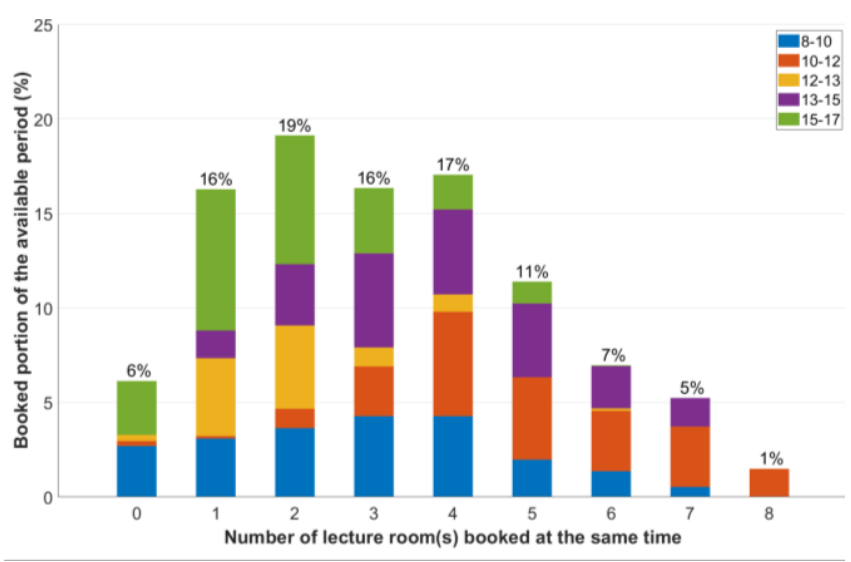

Figure 5. Aggregated analysis of all investigated lecture rooms

Time slot " $15-17$ " is rather under-utilized as never more than 5 lecture rooms were booked at the same time during that time slot. This indicates the probable viability to close down at least one lecture room by moving the lectures to other time slots. However, there is a need for further analysis to determine which one of the 8 lecture rooms should be chosen to close down. As all the lecture rooms have similar equipment for teaching and presentation, the size of lecture rooms are considered the main factor determining the preference of the staff on their choice of booking a certain lecture room. This implies the necessity to perform the analysis separately for each size category. Figure 6Error! Reference source not found. presents the results of this analysis for different size of lecture rooms. In about 13\% of the available periods all the 3 small lecture rooms were occupied at the same time while this proportion is $10 \%$ for the medium lecture rooms. The results also show in $21 \%$ of the available periods all the large lecture rooms were occupied at the same time although there are fewer large lecture rooms (2 lecture rooms) compared to the other sizes. As there are time slots when all the different categories of rooms are booked at the same time, it may create a problem on room availability on particular time slots if it is decided to close down a lecture room from each size. 
In the scenario to close down one of the lecture rooms while there is a shortage lecture room of the same size, the staff have several options including:

- booking a lecture room of the same size in the same day in a different time slot

- booking a lecture room of the same size in a different day

- booking a lecture room of different size in same day and same time slot

The analysis in this paper is limited to the possibility to book a lecture room of different size in same day and same time slot. This option might be desired by the staff who do not have the flexibility to change the schedule for their lecture or meeting.

\subsubsection{Booking a lecture room of different size}

Normally the alternative to the intended lecture room while a lecture room of the same size is not available would be to have a lecture room of different size which can accommodate all the occupants. For example, when staff want to book a small lecture room but it is not available, then they can instead book a medium or large lecture room. The possibility to replace the intended lecture room with a smaller lecture room depends on whether or not the staff choose the size of the lecture rooms optimally for the right number of attendees. Choosing a one size larger lecture room might associate with higher energy use although the savings achieved by closing down a lecture room may compensate it. Figure 7 shows the possibility to book a larger lecture room by examining the overlap between the times when all lecture rooms of the same size are booked and the time when one size larger lecture room is not available. The analysis is performed for different time slots and for 3 different scenarios including:

- When one small lecture room is closed down

- When one medium lecture room is closed down

- When one small and one medium lecture room is closed down

According to the last grouped bars (total) shown in Figure 7 , at $67 \%$ of the duration when all 3 small lecture rooms are booked, there is at least one medium lecture room empty and available to book. This means during the $13 \%$ of the total available time that 3 small lecture rooms are required, if one small lecture room is closed down, the staff have $67 \%$ chance to instead book a medium lecture room. However, at about 32 hours during one semester, there would not be a medium lecture room in the same time slot to replace the intended small lecture room.
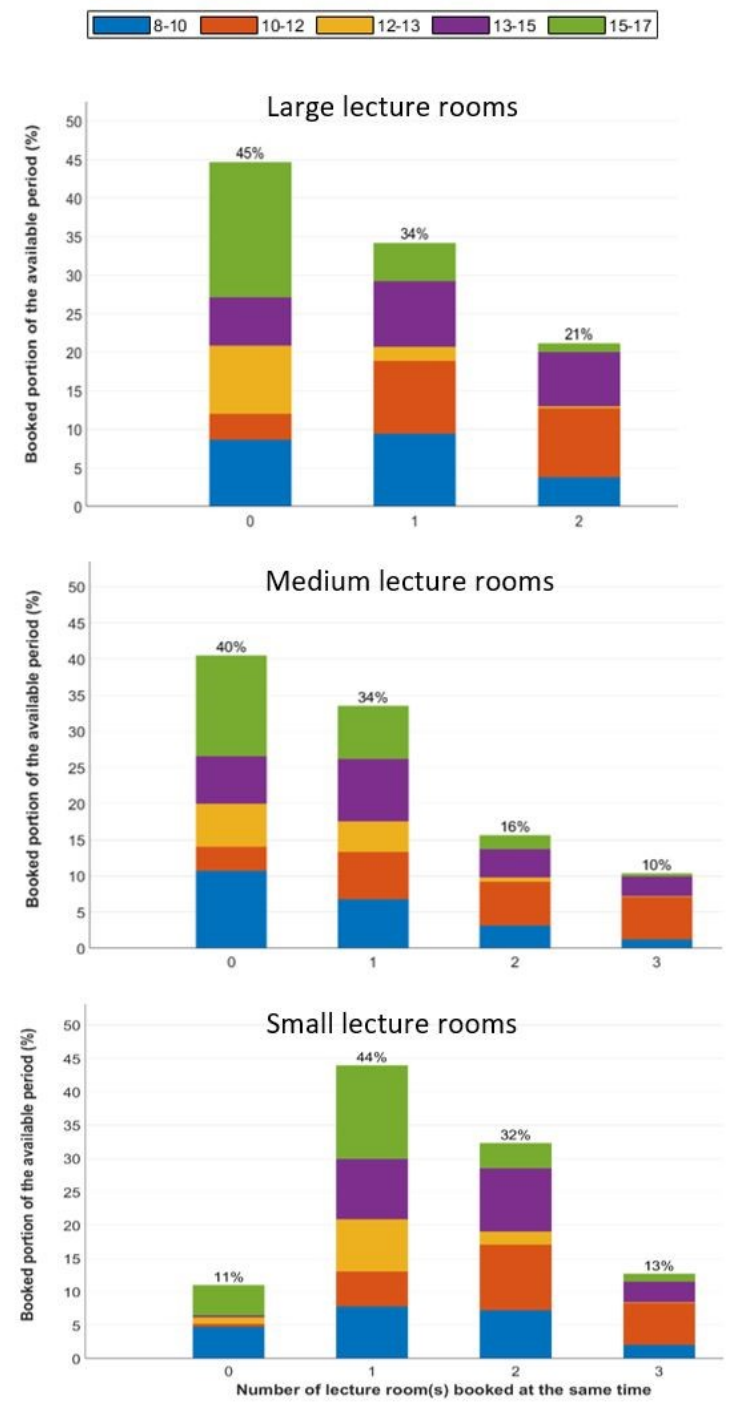

Figure 6. Aggregated analysis for different size of lecture rooms

Similar analysis for medium lecture rooms show that, if one medium lecture room is closed down, the staff have $60 \%$ chance to book a large lecture room when all the medium lecture rooms are booked while this may not be possible at 31 hours during a semester. Even in the scenario when two lecture rooms are closed down (one small and one medium lecture room), $45 \%$ of the times when two small lecture rooms are booked, there is at least 1 medium lecture room available. However, this option would not be available in 55 hours in a semester. The analysis for different time slots for this scenario shows the room shortage is significantly more likely to happen at "10-12". During this time slot, there are 23 hours that booking a one size larger lecture room is not possible when a small or medium lecture room is closed down. The shortage is exacerbated when one small and one medium lecture room are closed down simultaneously. There are 34 hours of unavailability of medium lecture rooms while all both small lecture rooms are booked. 


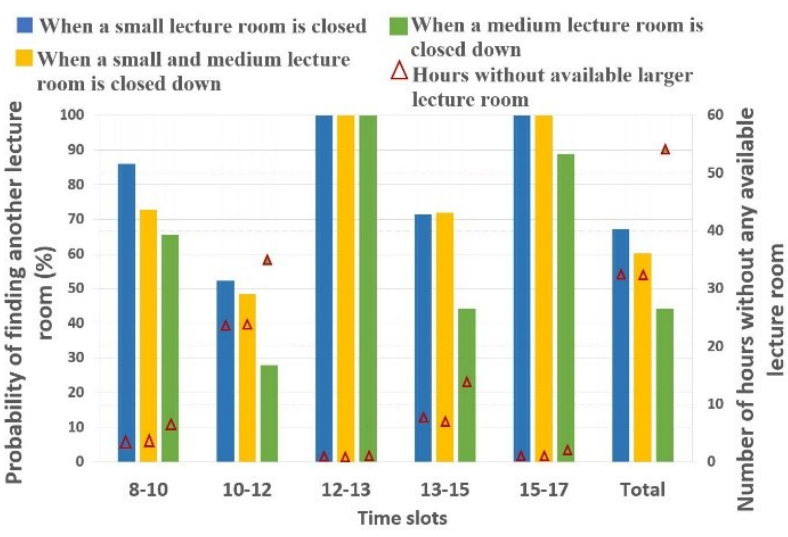

Figure 7. The possibility to book an alternative (larger) lecture room while closing down one or two lecture rooms

\section{Discussion}

Comprehensive space use assessment becomes possible within a smart campus, which is equipped with smart tools. Smart solutions such as large scale connected occupancy sensors and booking system can serve as the catalyst to transform insights on space use into practical application. Without the ability to monitor all lecture rooms within a building in a HEI at every moment, the space management decisions may be uninformed and ineffective. Smart tools in an IoT environment enable enhanced periodic space use reporting that offer potential for management interventions to optimize space and energy management. The information generated by smart tools can be used by universities to self-monitor and assist the decision makers to make informed choices to improve the effectiveness of resource allocation and environmental sustainability.

Analysing the data from occupancy sensors and booking systems in the 8 lecture rooms in a building in Umeå University revealed that they are highly underutilized. Moreover, the MPOR parameter showed the lecture rooms are not used spatially efficient in unbooked-occupied status. Limiting access to the lecture rooms for students in the periods that the lecture rooms are not booked may at least save the lighting energy. Moreover, the energy reduction for the ventilation supply can be achieved by implementing a ventilation system that operate based on occupancy. Such system can use the PIR sensors to infer the MPOR parameter for an approximate quick response to the number of occupants while fine-tuning the control settings based on $\mathrm{CO}_{2}$ concentration.

Closing down at least one lecture room ( 7 rooms instead of 8) seems to be effective to increase the booked proportion of lecture rooms during total time. The results indicate that there are only a few hours during a semester that it is not possible for the staff to book the next higher size larger lecture rooms when one of the lecture room is closed down. During those periods there are a few other options (see section 4.3) although this study does not particularly investigate them.
The energy saving potential of closing down each lecture room is estimated and presented in Table 2 considering the average energy use in university buildings in Sweden which is $201.4 \mathrm{kWh} / \mathrm{sqm}$-yr. As per the booking records, S1 and M3 lecture rooms are relatively less preferred rooms (Figure 2) and may be considered for closing down from small and medium size categories, respectively. Closing down both of them can potentially lead to a savings of approximately 19300 $\mathrm{kWh}$ per year besides the cost savings related to the housekeeping.

Table 2. Energy saving potential of closing down different lecture rooms

\begin{tabular}{|c|c|}
\hline Lecture room & Energy saving potential (kWh/year) \\
\hline S1 & 7250 \\
\hline S2 & 5030 \\
\hline S3 & 6850 \\
\hline M1 & 13090 \\
\hline M2 & 12080 \\
\hline M3 & 12080 \\
\hline
\end{tabular}

The booking system that incorporates information such as historical booking, occupancy information and energy use aspects can play a role in optimizing the space and energy use by providing information for selecting lecture rooms.

\section{Conclusion}

The lecture rooms in the investigated building at Umeå University that were found underused in our study provide opportunity to improve space use efficiency to reduce costs and environmental footprint. The nonintrusive data collection that is undertaken in this study is important for future expansion plans and the new knowledge about usage of different lecture rooms is already being discussed for interventions. The collected data in this study has only local relevance and may not be generalizable to other places however, the methods are applicable to other similar cases. This feasibility study proves that this model of space use monitoring is viable and useful to implement in other universities and organizations for space management and optimization.

The collected data in this study is related to only one semester although as suggested by [20], in order to reduce $m_{2}$ footprint, it is better to wait until a smart tool is implemented for an extended period of time. Further studies should also include different stakeholders by investigating the perceptions and preferences of staff and students on booking lecture rooms.

The authors gratefully acknowledge the H2020-SCC-2016 project nr 731198, RUGGEDISED, and the E2B2 program and the Swedish Energy agency for project nr 47878-1, Energy efficiency improvements in HVAC of University buildings by studying the occupancy pattern, for funding this work. 


\section{References}

1. Atzori L, Iera A, Morabito G. The Internet of Things: A survey. Comput Networks 2010;54:2787-805.

doi:https://doi.org/10.1016/j.comnet.2010.05.010

2. Elsaadany A, Soliman M. Experimental Evaluation of Internet of Things in the Educational Environment. Int J Eng Pedagog 2017;7:50. doi:10.3991/ijep.v7i3.7187.

3. Valks B, Arkesteijn MH, Den Heijer AC, Vande Putte HJM. Smart campus tools - adding value to the university campus by measuring space use real-time. J Corp Real Estate 2018;20:103-16. doi:10.1108/JCRE-03-2017-0006.

4. Rahman MSA, Ali HM, Sipan I, Awang M, Mohammed AH. Space utilization model for higher education institutions. $\mathrm{J}$ Teknol 2015;75:163-70. doi:10.11113/jt.v75.5285.

5. WHED. No 2019. https://whed.net/article.php?page=About (accessed December 24, 2019).

6. Trow MA. Reflections on the Transition from Elite to Mass to Universal Access: Forms and Phases of Higher Education in Modern Societies since WWII 2005.

7. Kuntz AM, Petrovic JE, Ginocchio L. A Changing Sense of Place: A Case Study of Academic Culture and the Built Environment. High Educ Policy 2012;25:433-51. doi:10.1057/hep.2011.29.

8. Ibrahim I, Yusoff WZW, Bilal K. Space Management: A Study on Space Usage Level in Higher Education Institutions. Procedia - Soc Behav Sci 2012;47:1880-7. doi:https://doi.org/10.1016/j.sbspro.2012.06.917.

9. eia. International Energy Outlook 2019 with projections to 2050. Washington: 2019.

10. Akademiska Hus. Annual and Sustainability Report 2018. Gothenburg: 2019.

11. Chung MH, Rhee EK. Potential opportunities for energy conservation in existing buildings on university campus: A field survey in Korea. Energy Build 2014;78:176-82. doi:10.1016/j.enbuild.2014.04.018.

12. Tu K-J, Lin C-H. Benchmarking energy efficiency by "space type": An energy management tool for individual departments within universities. J Asian Archit Build Eng 2012;11:299-306. doi:10.3130/jaabe.11.299.

13. Emeakaroha A, Ang CS, Yan Y, Hopthrow T. Integrating persuasive technology with energy delegates for energy conservation and carbon emission reduction in a university campus. Energy 2014;76:357-74. doi:https://doi.org/10.1016/j.energy.2014.08.027.

14. Johnes J. Operational Research in education. Eur J Oper Res 2015;243:683-96. doi:10.1016/j.ejor.2014.10.043.

15. Prentice KA, Argyropoulos EK. Library Space: Assessment and Planning through a Space
Utilization Study. Med Ref Serv Q 2018;37:13241. doi:10.1080/02763869.2018.1439213.

16. Knapp C, Vickroy K, De Bruyn L, Kwong D. Are the myths of space utilization costing you more than you know? J Corp Real Estate 2009; 11:237-43.

doi: $10.1108 / 14630010911006729$.

17. Space Management Group (2006). Space utilisation : practice, performance and guidelines. vol. 1. 2006.

18. Masoso OT, Grobler LJ. The dark side of occupants' behaviour on building energy use. Energy Build 2010;42:173-7.

19. Li N, Becerik-Gerber B. Performance-based evaluation of RFID-based indoor location sensing solutions for the built environment. Adv Eng Informatics 2011;25:535-46. doi:https://doi.org/10.1016/j.aei.2011.02.004.

20. Valks B, Arkesteijn M, Den Heijer A. Smart campus tools 2.0 exploring the use of real-time space use measurement at universities and organizations. Facilities 2019;37:961-80. doi:10.1108/F-11-2018-0136.

21. Rymarzak M, Marmot A. Higher Education Estate Data Accountability: The Contrasting Experience of UK and Poland. High Educ Policy 2018. doi:10.1057/s41307-018-0109-5.

22. Georgiou O, Raza U. Low Power Wide Area Network Analysis: Can LoRa Scale? IEEE Wirel Commun Lett 2017;6:162-5. doi:10.1109/LWC.2016.2647247. 